Case Report

\title{
Neuroleptic Malignant Syndrome, a Sequalae of Multiple Administration of Antipsychotics: A Case Report
}

\author{
Nervana Abdelfattah Hafez Elbakary ${ }^{*}$, Mohamed Adil Shah Khoodoruth \\ Mental Health Hospital, Hamad Medical Corporation, Doha, Qatar \\ Email address: \\ drnervanahafez@gmail.com (N. A. H. Elbakary), MKhoodoruth@hamad.qa (M. A. S. Khoodoruth) \\ ${ }^{*}$ Corresponding author
}

\section{To cite this article:}

Nervana Abdelfattah Hafez Elbakary, Mohamed Adil Shah Khoodoruth. Neuroleptic Malignant Syndrome, a Sequalae of Multiple Administration of Antipsychotics: A Case Report. American Journal of Psychiatry and Neuroscience. Vol. 7, No. 4, 2019 , pp. 83-87. doi: $10.11648 /$ j.ajpn.20190704.12

Received: September 21, 2019; Accepted: October 9, 2019; Published: November 5, 2019

\begin{abstract}
Neuroleptic malignant syndrome (NMS) is a lethal adverse drug reaction (ADR) that is often attributed to the administration of dopamine blockers, antiemetic agents as well as anti-parkinsonism medication withdrawal. We describe a challenging case of NMS, with malignant catatonia as a differential diagnosis, who was difficult to respond to treatment. The patient developed severe complications following her exposure to rapid titration using high doses of olanzapine, which was abruptly converted to chlorpromazine due to lack of efficacy, in addition to administering multiple as needed (PRN) medications for agitation. Hyperthermia, muscle rigidity, dysautonomia, mental status changes, catatonia, and some laboratory derangement were reported in this case. It is possible that factors such as naïve patients, high doses of neuroleptics, a switch from one agent to another, rapid escalation, and the increased use of parenteral therapy for managing of agitation acted collectively or individually as risk factors for developing NMS. Health care professionals should be cautious about the cumulative dose of antipsychotics used per day, including PRN medications. The delay in recognizing the early symptoms of NMS could put patients at higher risk of mortality. Lastly, it is pivotal to exclude other infectious or autoimmune etiologies before treating as NMS case.
\end{abstract}

Keywords: Malignant, Adverse Drug Reaction (ADR), Dopamine Blockers, Hyperthermia, Catatonia, Neuroleptics

\section{Introduction}

Neuroleptic malignant syndrome (NMS) is a lethal adverse drug reaction that results from the use of neuroleptics. NMS is defined by its association with a class of medications that block dopamine transmission and a myriad of distinctive clinical features, namely: hyperthermia, rigidity, mental status changes, and autonomic dysfunction. Laboratory derangement often reflects severe rigidity leading to more profound creatine kinase (CK) elevation and other common but non-specific findings such as leukocytosis, myoglobinuria, and electrolyte abnormalities. Epidemiological studies show that the incidence rate might range from $0.02 \%$ to $3 \%$ with a mortality rate of 55\% among all patients taking neuroleptics [1]. It is possible that certain factors such as naïve patients, higher doses of neuroleptics, a switch from one agent to another, rapid escalation, and the increased use of parenteral therapy for managing of agitation acted collectively or individually as risk factors for developing NMS [1-3]. The association of the syndrome with drug use is idiosyncratic. In other words, it is not a dose-dependent phenomenon, but higher dose act reliably as a risk factor [4].

Due to the complexity of elucidating a clear mechanism of NMS, some hypotheses have been put forward. Yamawaki et al proposed that under normal conditions where there is a balance between dopamine (DA) and serotonin (5-HT), DA acts to reduce body temperature and 5-HT acts to elevate body temperature $[5,6]$. The balance is easily disturbed as a result of dehydration and the additional administration of dopamine blocking agent.; Consequently, 5-HT becomes dominant and body temperature tends to rise. According to this hypothesis, NMS may occur.

Every class of neuroleptics, as well as all other drugs that affect the central dopaminergic system; have been implicated 
in developing NMS; either the first generation (e.g, chlorpromazine, haloperidol, fluphenazine) or the second generation (e.g, olanzapine, clozapine, risperidone) [7-10].

The objective of this paper is to demonstrate a challenging case of NMS, with malignant catatonia as differential diagnosis, who was difficult to respond to regular treatments, in addition to a relevant literature review of NMS cases.

\section{Case Presentation}

A 38-year-old Asian lady, previously healthy with no known drug allergies, was admitted to an inpatient unit in a mental health hospital as a case of brief psychotic disorder after presenting to the Emergency Department (ED) with insomnia, paranoid thoughts, and disorganized behavior. This was her second presentation to the ED. Her first presentation was one day earlier for panic attacks following a road traffic accident. Head Computed Tomography (CT) and urine drug screen were done in the ED with unremarkable results. The patient is married with three children and has no family history of mental illness.

Initially, she has prescribed a regular dose of olanzapine 10 $\mathrm{mg}$ bedtime, which was increased to $30 \mathrm{mg}$ on her second day of admission as she was disinhibited, responding to internal stimuli, and disoriented to time, but oriented to person and place. As part of her PRNs medications (as needed) for disruptive and violent behavior, she received $10 \mathrm{mg}, 15 \mathrm{mg}$, and $20 \mathrm{mg}$ of intramuscular haloperidol on the first, second, and fourth day of admission respectively, in addition to intramuscular Olanzapine $10 \mathrm{mg}$ as PRN on the second day. On the fifth day, Valproic Acid $500 \mathrm{mg}$ twice daily was prescribed for labile mood and disorderly behavior with nursing staff and other patients. On the sixth day, Chlorpromazine $400 \mathrm{mg}$ was ordered, and Olanzapine was discontinued in view of the impression that she was unable to sleep, restless, irritable, and agitated despite receiving $30 \mathrm{mg}$ of Olanzapine and $150 \mathrm{mg}$ of intramuscular diphenhydramine as PRN. On the ninth day, Chlorpromazine was increased to $500 \mathrm{mg}$ daily.

On the twelfth day, the psychiatry treating team consulted general medicine because of stupor, temperature of 38.4 degrees Celsius, and white cell count of 12,100/microliter with a shift to the left (neutrophil count 10,300/microliter). An electrocardiogram revealed no acute ischemic changes. Septic workup was initiated along with intravenous fluids for presumed urinary tract infection. Laboratory investigations revealed CRP $68 \mathrm{mg} / \mathrm{l}$, lactic acid $1.2 \mathrm{mmol} / \mathrm{L}$, procalcitonin $0.04 \mathrm{ng} / \mathrm{ml}$, CK $590 \mathrm{U} / \mathrm{L}$, myoglobin $158 \mathrm{ng} / \mathrm{ml}$, and normal liver enzymes. Chest X-ray was unremarkable. Additionally, chlorpromazine was tapered off and intramuscular Lorazepam $2 \mathrm{mg}$ twice daily was ordered. On day fifteen, she began to have severe rigidity in all limbs and altered consciousness. All antipsychotics were discontinued, and she was kept on intramuscular lorazepam $2 \mathrm{mg}$ twice daily. On the eighteenth day, she was transferred to the medical floor because of intermitted spikes of fever (38.8 degrees Celsius), tachycardia (HR120 beat/minute), elevated blood pressure (169/108
$\mathrm{mmHg}$ ), persisted altered level of consciousness, along with disorientation to person, time and place.

Laboratory and radiological studies revealed white cell count of $10,700 /$ microliter with a shift to the left (neutrophil count 7,800/microliter), myoglobinuria, negative blood cultures, negative urine toxicology screen except positive for benzodiazepine, and no signs of acute intracranial abnormality on CT Head. Urinalysis was positive for nitrite with a negative urine culture. Fluoroscopic-guided lumbar puncture revealed WBC 17 /ul, 97\% lymphocytes, RBC 1962 /ul, glucose 3.72 $\mathrm{mmol} / \mathrm{l}$, protein $0.30 \mathrm{~g} / \mathrm{L}$, with negative culture and negative acid-fast bacilli and TB PCR. viral panel was also negative.

The psychiatry consultation-liaison team followed the patient on the medical floor and their impression was neuroleptic malignant syndrome (NMS). The patient also had features of catatonia such as mutism, posturing, stupor, and waxy positioning, therefore, lorazepam was increased to $2 \mathrm{mg}$ three times a day. The medical team started her on intravenous dantrolene for nine days. They also shifted the patient to the medical intensive care unit (MICU) and consulted Neurology. On the twenty-second day, the nursing staff noted face twitching, neck spasm, and tonic-clonic movements which aborted by intravenous midazolam $5 \mathrm{mg}$. The following day she was started on intravenous levetiracetam $500 \mathrm{mg}$ twice daily because of intermittent tonic seizures; it was further increased to 1 gram twice daily on the twenty-third day.

On the twenty-fourth day, the magnetic resonance imaging (MRI) with contrast, requested by Neurology, suggested no findings of meningitis or encephalitis. Electroencephalogram (EEG) showed generalized slowing with no epileptic discharges, and no increased intracranial pressure (ICP). Additional blood work revealed leukocytosis - WBC 21,300/ microliter with a shift to the left (neutrophil count 19,000/microliter), CK $742 \mathrm{U} / \mathrm{L}$, serum potassium $3.3 \mathrm{mmol} / \mathrm{L}$, total protein $60 \mathrm{~g} / \mathrm{L}$ with $26.6 \mathrm{~g} / \mathrm{L}$ of albumin, ALT $132 \mathrm{U} / \mathrm{L}$, and AST $116 \mathrm{U} / \mathrm{L}$. Autoimmune markers negative for ANCA, Anti Cardiolipin Antibody $\operatorname{IgG}$ and IgM, Antithyroid Peroxidase, and Antithyroglobulin Antibody. General Immunology workup showed $\mathrm{IgA}, \mathrm{C} 3$, and $\mathrm{C} 4$ to be within normal limits. Neurology suspected autoimmune encephalitis as a probable differential and empirically started the patient on Intravenous Immunoglobulin (IVIG) $0.4 \mathrm{gm} / \mathrm{kg} / \mathrm{day}$ for five days and on continuous EEG for 3 days. Malignant catatonia was also challenging in their differential diagnosis. In view of deteriorating liver function tests (LFTs), dantrolene was discontinued, and LFTs normalized thereafter. The medical team ordered pan-Computed Tomography which revealed features of aspiration pneumonia (for which she received intravenous Piperacillin-Tazobactam $4 \mathrm{~g} / 0.5 \mathrm{~g}$ three times daily for three days) and hypodense nodule in the left thyroid lobe.

By day thirty, the patient was seizure-free for five days except for occasional lip smacking and bilateral arm tremors, however, she was still experiencing high fever and blood pressure fluctuation. Continuous EEG monitoring revealed no epileptic occurrences. Thus, Neurology tapered off levetiracetam and PRN labetalol was ordered. The medical team started her on Bromocriptine $2.5 \mathrm{mg}$ three times a day. 
On day thirty-five, the patient was stepped down from the MICU to the medical floor for supportive care and physiotherapy. The psychiatric treating team noted some improvement in communication and sensorium, and no catatonic symptoms could be elicited. By day forty, she started speaking coherently, relevantly and obeyed commands. Her Foley's catheter and Nasogastric Tube were also removed. She was referred to the inpatient rehabilitation program. She was discharged on day forty-five, and no follow-up could be done thereafter as she traveled with her family permanently from the country.

A written informed consent was signed by the patient before discharge, and institutional approval letter was obtained to allow authors to publish this case report.

A timeline chart summarized the patient's journey across multiple sites in Figure 1.

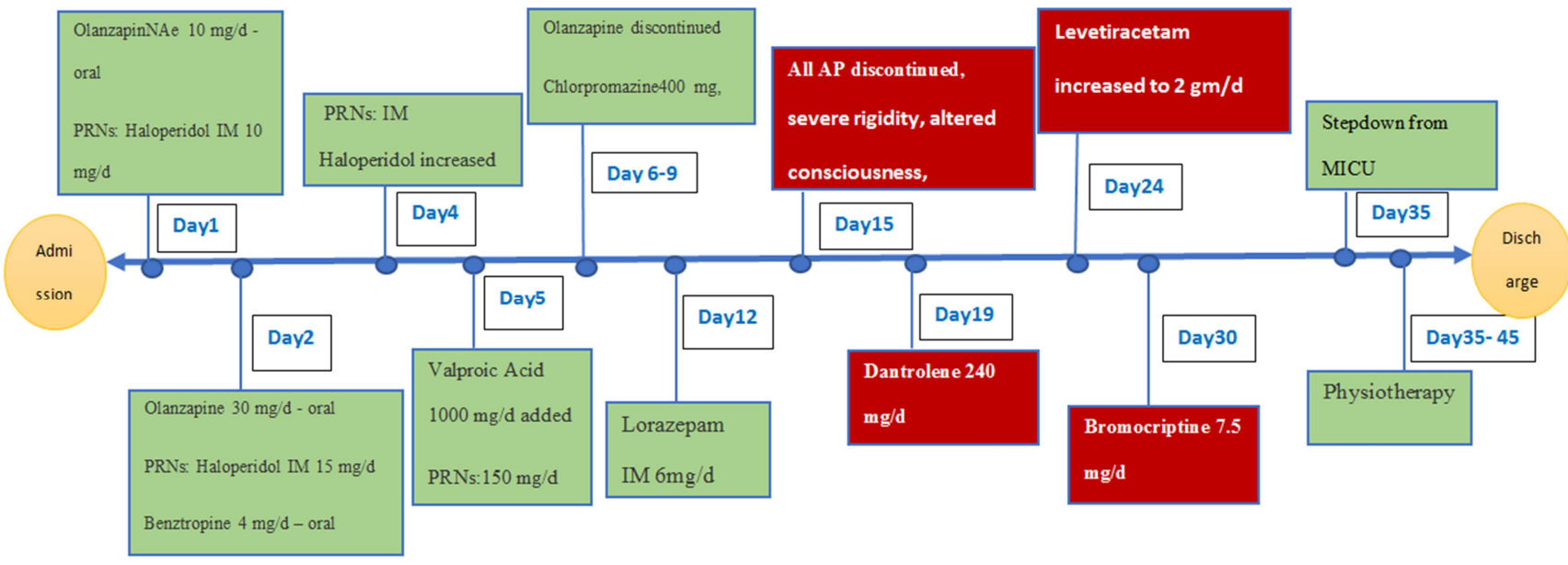

Inpatient unit - psychiatry hospital

Figure 1. Timeline chart of the NMS case report

AP: antipsychotics; IM: intramuscular; IVIG: intravenous immunoglobulin; mg/d: milligram/day; MICU: medical intensive care unit; PRN: as needed medication.

\section{Discussion}

A systematic review of the literature was conducted by searching the PUBMED, MEDLINE, Google Scholar, Science OPEN databases on January 29, 2019, using the following keywords: (Neuroleptic malignant syndrome OR Malignant hyperthermia OR Fever induced) AND (antipsychotic OR neuroleptic).

Inclusion criteria were all types of clinical research papers in English. Exclusion criteria were non-clinical research papers or papers published in languages other than English. Preliminary search sorted by best match yielded a total of 11312 results. After we filter for free full texts, adults, 609 results identified. After removal of duplicates, title, and abstract analysis, only 21 relevant papers were retained with a total of 89 patients who therefore included in the review.

Like many other patients who had developed neuroleptic malignant syndrome (NMS), our case combined typical features of NMS. Disturbances in consciousness level appeared in $82 \%$ of the studied patients [11]. It is not surprising, given the usual psychiatric comorbidity of the typical patient, that its implication is often underrecognized. It often takes the form of an agitated delirium with confusion rather than psychosis. Muscular rigidity and superimposed tremor may lead to a ratcheting quality or a cogwheel phenomenon (seen in 45 to $92 \%$ )-[12, 13]. Hyperthermia after administration of antipsychotic drugs is a defining symptom according to many diagnostic criteria. Temperature of more than $38^{\circ} \mathrm{C}$ was seen in $87 \%$ of patients [12], but even higher temperatures, greater than $40^{\circ} \mathrm{C}$, are common [13]. Dysautonomia takes the form of tachycardia (in $88 \%$ ), labile or high blood pressure (in 61 to $77 \%$ ), and tachypnea (in 73\%) $[12,14]$. Dysrhythmia could also occur.

$70 \%$ of patients followed a typical course of mental status changes appearing first, followed by rigidity, then hyperthermia, and autonomic dysfunction [11].

We attributed the induction of NMS in this case to either olanzapine and/or chlorpromazine. Olanzapine was mentioned in the literature in 31 case reports as the main factor or a co-factor with other agents for developing NMS [9, 15], $[16,17]$. Chlorpromazine was involved in 8 studies $[12,18]$.

The management of the psychotic symptoms related to this patient is considered one of its limitations. Sachdev et al found in a case-control study that NMS is more likely to occur in a patient who is in agitated or dehydrated state, often needing restraint or seclusion, as well as who receives higher doses of neuroleptic medication soon after admission to hospital, and who continues to receive high doses over the following few days [19]. This is evident in our case report as the patient was treated with a rather high escalating dose of olanzapine (up to $30 \mathrm{mg}$ daily) followed by an abrupt change to chlorpromazine (up to $500 \mathrm{mg}$ daily), along with administering multiple PRNs, 
which undoubtedly compounded the antipsychotic burden in a short period of time, thus synergistically flaring up the neuroleptic side effects.

The strong point, in this case, was in the differential diagnosis because NMS is a diagnosis of exclusion. In addition to thyrotoxicosis and sepsis, which could be excluded via the laboratory studies, we should take Central Nervous System (CNS) infections into consideration, especially viral encephalitis, which can be hard to distinguish from NMS. According to negative results of cerebral spinal fluid (CSF) studies, electroencephalography and neuroimaging, the possibility of NMS is high. Malignant catatonia could act as a life-threatening manifestation which can occur in the context of an underlying neuropsychiatric illness and shares clinical manifestations and medical prognosis with the Neuroleptic Malignant Syndrome (NMS).

MOdified NARanjo Causality Scale for an Individual case safety report - (MONARCSI) is a tool that assists safety professionals performing causality assessments between an identified untoward clinical event and a drug using a simple questionnaire to assign probability scores. Authors applied the scale, and it yielded a score of 15.05 which was read as a high predictive value indicating highly related causality between both olanzapine / chlorpromazine and NMS [20]. Severity was evaluated using Hartwig's Severity Assessment Scale and the output was level 5, which interpreted to severe reaction. Another specific NMS rating scale was developed by Sachdev et al and her score was 22/36 which meant: definite NMS [21].

\section{Conclusion}

We reported a refractory case that developed severe complications of Neuroleptic Malignant Syndrome (NMS) with malignant catatonia as differential diagnosis following her exposure to multiple antipsychotics. Malignant catatonia could act as a life-threatening manifestation which can occur in the context of an underlying neuropsychiatric syndrome and shares clinical and pathophysiological features and medical prognosis with the Neuroleptic Malignant Syndrome (NMS). Health care professionals should be cautious about the cumulative dose of antipsychotics used per dayincluding PRN or as needed treatments- for management of acute agitation. It is essential to rule out other infectious or autoimmune etiologies. Authors advocate using rating scores as helpful tools capable of giving a comprehensive evaluation for causality and severity of the related adverse drug reactions.

\section{References}

[1] S. Modi, D. Dharaiya, L. Schultz, and - Panayiotis Varelas, "Neuroleptic Malignant Syndrome: Complications, Outcomes, and Mortality," Neurocrit Care, no. 24, pp. 97-103, 2016.

[2] D. Berardi, M. Amore, P. E. Keck, M. Troia, and M. Dell'Atti, "Clinical and pharmacologic risk factors for neuroleptic malignant syndrome: a case-control study.," Biol. Psychiatry, vol. 44, no. 8, pp. 748-54, Oct. 1998.

[3] P. E. Keck, H. G. Pope, B. M. Cohen, S. L. McElroy, and A. A. Nierenberg, "Risk factors for neuroleptic malignant syndrome. A case-control study.," Arch. Gen. Psychiatry, vol. 46, no. 10, pp. 914-8, Oct. 1989.

[4] J. Langan, D. Martin, P. Shajahan, and D. J. Smith, "Antipsychotic dose escalation as a trigger for neuroleptic malignant syndrome (NMS): literature review and case series report.," BMC Psychiatry, vol. 12, no. 1, p. 214, Nov. 2012.

[5] H. A. Yamawaki S, Lai H, "Dopaminergic and serotonergic mechanisms of thermoregulation: mediation of thermal effects of apomorphine and dopamine," J. Pharmacol. Exp. Ther., vol. 227, no. Issue 2, pp. 383-388, 1983.

[6] G. Northoff, "Catatonia and neuroleptic malignant syndrome: psychopathology and pathophysiology," J. Neural Transm., vol. 109, no. 12, pp. 1453-1467, Dec. 2002.

[7] G. J. Chandran, J. R. Mikler, and D. L. Keegan, "Neuroleptic malignant syndrome: case report and discussion.," CMAJ, vol. 169, no. 5, pp. 439-42, Sep. 2003.

[8] D. P. Seitz and S. S. Gill, "Neuroleptic Malignant Syndrome Complicating Antipsychotic Treatment of Delirium or Agitation in Medical and Surgical Patients: Case Reports and A Review of the Literature," Psychosomatics, vol. 50, no. 1, pp. 8-15, Jan. 2009.

[9] A. Kogoj and I. Velikonja, "Olanzapine induced neuroleptic malignant syndrome?a case review," Hum. Psychopharmacol. Clin. Exp., vol. 18, no. 4, pp. 301-309, Jun. 2003.

[10] S. C. Stoner and A. Berry, "Suspected Neuroleptic Malignant Syndrome During Quetiapine-Clozapine Cross-Titration," J. Pharm. Pract., vol. 23, no. 1, pp. 69-73, Feb. 2010.

[11] V. R. Velamoor, R. M. Norman, S. N. Caroff, S. C. Mann, K. A. Sullivan, and R. E. Antelo, "Progression of symptoms in neuroleptic malignant syndrome.," J. Nerv. Ment. Dis., vol. 182, no. 3, pp. 168-73, Mar. 1994.

[12] J. L. Levenson, "Neuroleptic malignant syndrome," Am. J. Psychiatry, vol. 142, no. 10, pp. 1137-1145, Jun. 2006.

[13] S. N. Caroff and S. C. Mann, "Neuroleptic malignant syndrome.," Med. Clin. North Am., vol. 77, no. 1, pp. 185-202, Jan. 1993.

[14] P. Rosebush and T. Stewart, "A prospective analysis of 24 episodes of neuroleptic malignant syndrome.," $\mathrm{Am}$. J. Psychiatry, vol. 146, no. 6, pp. 717-25, Jun. 1989.

[15] M. S. Arnaout, F. P. Antun, and K. Ashkar, "Neuroleptic malignant syndrome with olanzapine associated with severe hypernatremia," Hum. Psychopharmacol. Clin. Exp., vol. 16, no. 3, pp. 279-281, Apr. 2001.

[16] S. C. Stanfield and T. Privette, "Neuroleptic malignant syndrome associated with olanzapine therapy: a case report.," $J$. Emerg. Med., vol. 19, no. 4, pp. 355-7, Nov. 2000.

[17] V. P. Kontaxakis, B. J. Havaki-Kontaxaki, N. G. Christodoulou, K. G. Paplos, and G. N. Christodoulou, "Olanzapine-associated neuroleptic malignant syndrome: Is there an overlap with the serotonin syndrome?," Ann. Gen. Hosp. Psychiatry, vol. 2, no. 1, p. 10, Oct. 2003. 
[18] T. Mahmood and J. P. Warren, "Neuroleptic malignant syndrome from chlorpromazine: case report.," J. R. Coll. Gen. Pract., vol. 39, no. 322, p. 211, May 1989.

[19] P. Sachdev, C. Mason, and D. Hadzi-Pavlovic, "Case-control study of neuroleptic malignant syndrome," Am. J. Psychiatry, vol. 154, no. 8, pp. 1156-1158, Aug. 1997.
[20] S. Comfort, D. Dorrell, S. Meireis, and J. Fine, "MOdified NARanjo Causality Scale for ICSRs (MONARCSi): A Decision Support Tool for Safety Scientists," Drug Saf., vol. 41, no. 11, pp. 1073-1085, Nov. 2018.

[21] P. S. Sachdev, "A rating scale for neuroleptic malignant syndrome. Psychiatry Research; 135: 249-256.," Psychiatry Res., vol. 135, pp. 249-256., 2005. 